\title{
O tratamento da Classe III - revisão sistemá- tica - Parte I. Magnitude, direção e duração das forças na protração maxilar
}

Anna Paula Rocha Perrone*, José Nelson Mucha**

\begin{abstract}
Resumo
Introdução: para o tratamento da Classe III durante o crescimento, a protração maxilar é a terapia mais relatada na literatura. Durante o tratamento, é requerida especial atenção à mecânica utilizada para a correção do problema. Objetivo: sintetizar as informações relativas à magnitude, direção e tempo diário da aplicação das forças, por meio de uma revisão sistemática. Métodos: estudos foram identificados a partir de uma pesquisa eletrônica no Medline database - Entrez PubMed (178 artigos) e Bireme (550 artigos), no período de janeiro de 1983 a dezembro de 2008. Após um criterioso processo de inclusão e exclusão, 56 estudos primários foram selecionados e submetidos a um segundo processo de seleção, restando 39 artigos. Foram calculados a média e o desvio-padrão, bem como os valores mínimos e máximos para a magnitude, a direção e a quantidade de horas de uso das forças de protração maxilar. Resultados e Conclusão: as médias de magnitude, direção e duração das forças de protração maxilar foram de, respectivamente, 447,8 gramas, 27,5 graus de inclinação em relação ao Plano Oclusal, e 15,2 horas por dia.
\end{abstract}

Palavras-chave: Má oclusão Classe III de Angle. Aparelhos ortopédicos. Ortodontia. Máscaras faciais.

\section{INTRODUÇÃO}

A etiologia multifatorial e a dificuldade de prever o padrão de crescimento do complexo craniofacial contribuem para que o tratamento da Classe III seja desafiador. Essa pode ser caracterizada pelo retrognatismo maxilar, prognatismo mandibular ou a combinação de ambos. Entretanto, a retrusão da maxila foi considerada a situação mais comum ${ }^{1,42}$. Uma pesquisa realizada em latinos ${ }^{36}$ mostrou sua incidência em $5 \%$ da população estudada, a qual variou de 3,3\% a 4,4\% na população brasileira ${ }^{7}$. Em estudos na população asiática, observou-se uma incidência maior, variando de $9 \%$ a $19 \%$, sendo sua prevalência em torno de $16,7 \%$ em pacientes coreanos $^{18}$.

Várias são as possibilidades de tratamento para a Classe III. Todavia, a maioria dos autores são unânimes em considerar a protração maxilar associada ou não à expansão palatina rápida como a melhor terapia para os pacientes em fase de crescimento $1,2,12,21,24,32,40,41,43$.

Diferentes tipos de dispositivos utilizados como ancoragem extrabucal para protração maxilar já foram descritos na literatura, como: máscara facial tipo Delaire, máscara facial tipo Petit, máscara facial de Turley e Sky Hook, entre outros ${ }^{2,21}$.

* Especialista em Ortodontia e Ortopedia Facial pela PUC-RJ.

** Doutor em Ortodontia e professor titular de Ortodontia na UFF, Niterói/RJ. 
Desde 1960, trabalhos têm sido realizados para verificar a possibilidade da movimentação da maxila. Em 1977, um estudo em macacos mostrou ser possível movimentar a maxila no sentido anterior por meio da aplicação de força extrabucal ${ }^{17}$.

A magnitude, a direção do vetor da força aplicada e a quantidade de horas de uso durante o dia são extremamente importantes para o sucesso da terapia com protração maxilar. Uma inclinação inferior a $20^{\circ}$ em relação ao plano oclusal promoveria uma rotação da maxila no sentido anti-horário. Para alguns autores, a direção da força deveria ser horizontal ou levemente inferior, de acordo com o grau de sobremordida do paciente. Estudos em crânio seco indicaram que uma força aplicada acima do plano de Frankfurt produz uma rotação posterior da maxila ${ }^{14}$. A força aplicada e a quantidade de horas de uso são um tema também controverso na literatura, pois existe um número grande de combinações entre a magnitude da força e o tempo de utilização do dispositivo.

Embora a literatura seja vasta em relação à terapia com protração maxilar, uma revisão sistemática de ensaios clínicos se faz necessária para sintetizar as informações referentes à magnitude da força, ao vetor de direção e ao período de uso diário do aparelho extrabucal. A revisão sistemática de ensaios clínicos é um método reprodutível, que apresenta critérios definidos de avaliação para a inclusão e a exclusão de estudos, sintetizando as informações para auxiliar na tomada de decisões clínicas $^{26}$, além de auxiliar na explicação de diferenças encontradas entre estudos primários que investigaram a mesma questão.

A única revisão sistemática, até então existente na literatura, sobre o tratamento da má oclusão de Classe III não avaliou diretamente as forças, os vetores de direção e as horas diárias de uso ideais para o tracionamento maxilar ${ }^{39}$.

O objetivo deste estudo é, portanto, por meio de uma revisão sistemática, analisar as evidências, revendo e sintetizando os estudos primários (ensaios clínicos) para obter informações sobre as médias e as variações relativas a: (1) magnitude da força utilizada para protração maxilar; (2) direção do vetor da força aplicada; e (3) quantidade de horas recomendada para o uso diário do aparelho de protração maxilar.

\section{MATERIAL E MÉTODOS}

A identificação dos estudos relacionados ao tratamento da Classe III foi realizada a partir de uma pesquisa computadorizada utilizando as seguintes fontes de informação:

- Medline database - Entrez PubMed (http:// www.ncbi.nlm.nih.gov/);

- Bireme (Centro Latino Americano e do Caribe de Informação em Ciências da Saúde): Lilacs, SciELO, Biblioteca Cochrane (http://www.bireme. br/php/index/php);

- Biblioteca Cochrane (http:www.bireme.br/ cochrane);

- Base de Dados Cochrane de Revisões Sistemáticas (The Cochrane Database of Systematic Reviews);

- Registro Cochrane de Ensaios Clínicos Controlados (The Cochrane Controlled Trials Register).

O levantamento abrangeu um período de 25 anos (janeiro de 1983 a dezembro de 2008).

No processo inicial de pesquisa no Medline database - Entrez PubMed foram utilizadas as palavras-chave "malocclusion" e "Class III", sendo identificados 1.819 artigos. Para filtrar a pesquisa, foram adicionadas as palavras-chave "treatment" e "early", sendo encontrados 178 artigos, conforme demonstrado na figura 1 e no quadro 1.

Durante o processo de pesquisa na base de dados da Bireme, foram utilizadas as palavras-chave "malocclusion" e "Class III", obtendo-se como resultado 2.403 trabalhos, sendo 2.341 artigos, 37 teses e 25 monografias.

Para filtrar a pesquisa, foi acrescentada a palavra chave "treatment", resultando em 591 trabalhos, sendo 570 artigos, 18 teses e 3 monografias.

Apenas os artigos foram considerados para o trabalho. Quando a pesquisa foi restringida para o 
período de janeiro de 1983 a dezembro de 2008, foram identificados 550 artigos, conforme apresentado na figura 1 e no quadro 1.

Os resumos dos artigos inicialmente selecionados nas bases de dados Medline database - Entrez PubMed (178 artigos) e Bireme (550 artigos) foram analisados. Foi realizada uma verificação para evitar artigos repetidos (que constavam na seleção das duas bases de dados). Após esse procedimento, os artigos passaram por um primeiro processo de inclusão e exclusão, conforme mostra o quadro 2.

A partir dessa primeira seleção, foram identi-

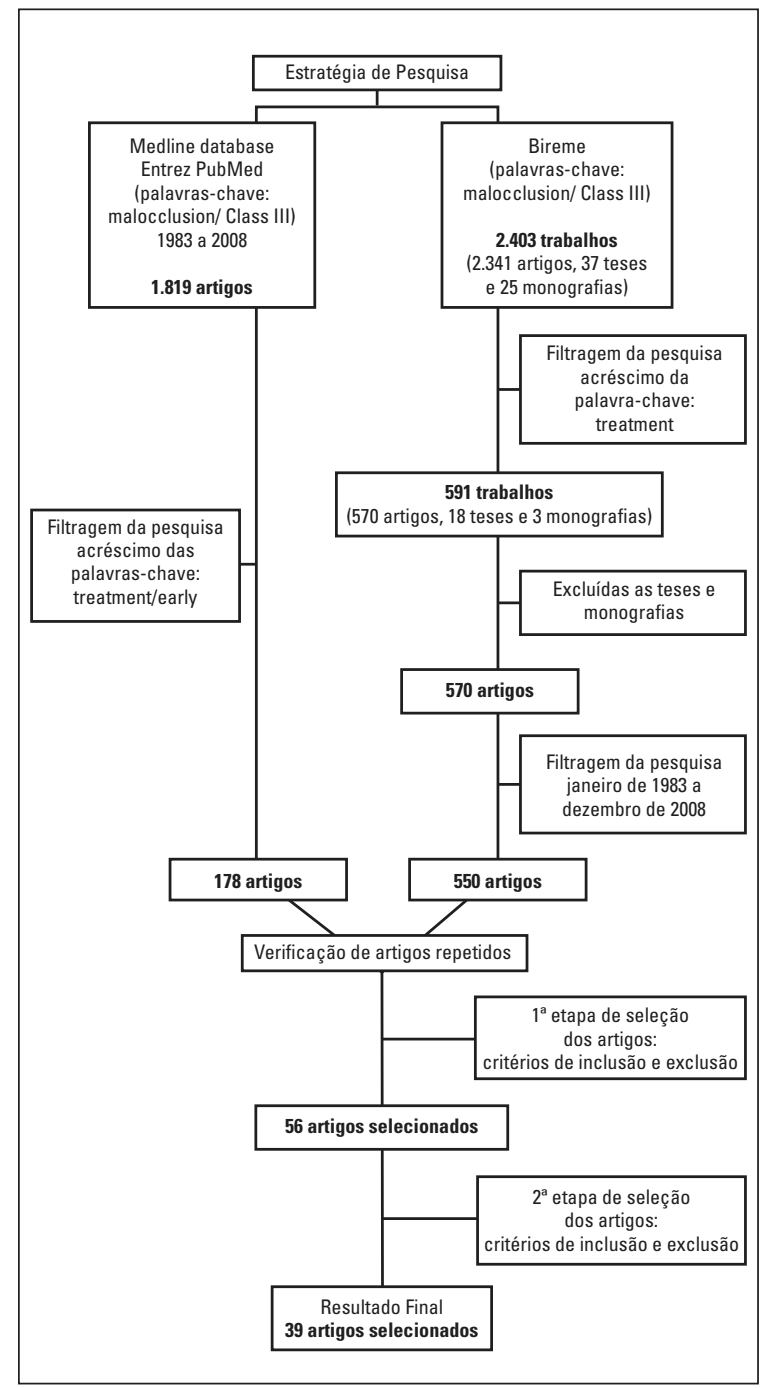

FIGURA 1 - Organograma demonstrando as estratégias de pesquisa.

ficados 56 estudos primários, dos quais 13 artigos constavam no Registro Cochrane de Ensaios Clínicos Controlados.

Esses 56 artigos pré-selecionados passaram por uma segunda fase de inclusão e exclusão, apresentada no quadro 3.

\begin{tabular}{cccc} 
PALAVRAS-CHAVE & $\begin{array}{c}\text { BASE DE } \\
\text { DADOS }\end{array}$ & PERÍODO & $\begin{array}{c}\text { RESULTADO DA } \\
\text { PESQUISA }\end{array}$ \\
$\begin{array}{c}\text { malocclusion. } \\
\text { Class III. }\end{array}$ & $\begin{array}{c}\text { Medline } \\
\text { (PubMed) }\end{array}$ & $1983-2008$ & 1.819 artigos \\
$\begin{array}{c}\text { malocclusion. } \\
\text { Class III. } \\
\text { treatment.early. }\end{array}$ & $\begin{array}{c}\text { Medline } \\
\text { (PubMed) }\end{array}$ & $1983-2008$ & 178 artigos \\
$\begin{array}{c}\text { malocclusion. } \\
\text { Class III. }\end{array}$ & Bireme & & $\begin{array}{c}2.341 \text { artigos } \\
37 \text { teses }\end{array}$ \\
$\begin{array}{c}\text { malocclusion. } \\
\text { Class III. } \\
\text { treatment. }\end{array}$ & Bireme & $1983-2008$ & 25 monografias \\
\hline
\end{tabular}

QUADRO 1 - Estratégia de busca e respectivos resultados.

CRITÉRIOS DE INCLUSÃO

I - Meta-análise, revisão sistemática, ensaios clínicos aleatórios, estudos prospectivos e retrospectivos

II - Artigos publicados de janeiro de 1983 até dezembro de 2008

III - Estudos com pacientes em fase de crescimento

IV - Estudos com protração maxilar associada ou não à expansão palatina rápida

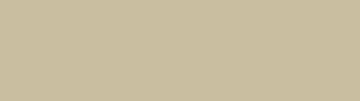

QUADRO 2 - Critérios de inclusão e exclusão da primeira fase de seleção.

\begin{tabular}{ll}
\multicolumn{1}{c}{ CRITÉRIO DE INCLUSÃO } & \multicolumn{1}{c}{ CRITÉRIO DE EXCLUSÃO } \\
Estudos com a terapia de & Estudos com a terapia de \\
protração maxilar, associada & protração maxilar, associada \\
ou não à expansão palatina & ou não à expansão palatina \\
rápida, que identificaram a & rápida, que não identifica- \\
magnitude, o vetor de direção & ram a magnitude, o vetor de \\
e o tempo diário da aplicação & $\begin{array}{l}\text { direção e o tempo diário da } \\
\text { das forças }\end{array}$ \\
aplicação das forças
\end{tabular}

QUADRO 3 - Critérios de inclusão e exclusão da segunda fase de seleção. 
TABELA 1 - Artigos selecionados após a segunda fase de inclusão e exclusão.

\begin{tabular}{|c|c|c|c|c|c|}
\hline ARTIGO & $n$ & APARELHO & FORÇA & $\begin{array}{l}\text { DIREÇÃO DO } \\
\text { PLANO OCLUSAL }\end{array}$ & $\mathbf{t}$ \\
\hline Oktay, Ulukaya ${ }^{31}$ & 20 & Máscara Tubinger/Petit & $600 \mathrm{~g}$ & $30^{\circ}$ & $16 \mathrm{~h}$ \\
\hline Kircelli, Pektas 22 & 6 & Miniplaca de titânio + Máscara Facial de Delaire & $300 \mathrm{~g}$ & $30^{\circ}$ & $22 \mathrm{~h}$ \\
\hline Kilinç et al. ${ }^{20}$ & 18 & Hyrax + Máscara Facial de Petit & $650 \mathrm{~g}$ & $20^{\circ}$ & $18 \mathrm{~h}$ \\
\hline Tortop et al. ${ }^{40}$ & 28 & Hyrax + Máscara Facial de Delaire & $600 \mathrm{~g}$ & $20^{\circ}$ & $16 \mathrm{~h}$ \\
\hline Pangrazio-Kulbersh et al. ${ }^{33}$ & 17 & Disjuntor Palatino + Máscara Facial & $500 \mathrm{~g}$ & $30^{\circ}$ & $15 \mathrm{~h}$ \\
\hline Yoshida et al. ${ }^{45}$ & 42 & Aparelho intrabucal + APM + Mentoneira & $250 \mathrm{~g}$ & $20^{\circ}$ & $14 \mathrm{~h}$ \\
\hline Arman et al. ${ }^{2}$ & 14 & Disjuntor Palatino + Máscara Facial & $500 \mathrm{~g}$ & $25^{\circ}$ & $14 \mathrm{~h}$ \\
\hline Kama, Özer, Baran ${ }^{16}$ & 15 & Ap. Removível + Máscara Facial de Delaire & $600 \mathrm{~g}$ & $20^{\circ}$ & $17 \mathrm{~h}$ \\
\hline Baccetti et al. ${ }^{5}$ & 32 & Disjuntor Palatino + Máscara Facial & $400 \mathrm{~g}$ & Frente e p/ baixo & $14 \mathrm{~h}$ \\
\hline Yoshida et al. ${ }^{46}$ & 32 & Aparelho intrabucal + APM + Mentoneira & $250 \mathrm{~g}$ & $20^{\circ}$ & $14 \mathrm{~h}$ \\
\hline Vaughn et al. ${ }^{41}$ & 29 & Aparelho de Expansão + Máscara Individual (Turley) & $400 \mathrm{~g}$ & $22,5^{\circ}$ & $22 \mathrm{~h}$ \\
\hline Cozza et al. ${ }^{9}$ & 30 & Aparelho intrabucal + Máscara Facial & $400 \mathrm{~g}$ & Frente e p/ baixo & $14 \mathrm{~h}$ \\
\hline Kajiyama et al. ${ }^{15}$ & 63 & Ap. Protração (MPBA) & $800 \mathrm{~g}$ & $30^{\circ}$ & $11 \mathrm{~h}$ \\
\hline Franchi et al. ${ }^{10}$ & 50 & Aparelho de Expansão + Máscara Facial & $400 \mathrm{~g}$ & Frente e p/baixo & $14 \mathrm{~h}$ \\
\hline Weissheimer et al. ${ }^{42}$ & 27 & Haas + Máscara Facial & $362,5 \mathrm{~g}$ & $20^{\circ}$ & $22 \mathrm{~h}$ \\
\hline Kyung-Suk ${ }^{23}$ & 85 & Hyrax + Máscara Facial de Delaire & $500 \mathrm{~g}$ & $30^{\circ}$ & $12 \mathrm{~h}$ \\
\hline Westwood et al. ${ }^{43}$ & 34 & Disjuntor Palatino + Máscara Facial & $400 \mathrm{~g}$ & Frente e p/ baixo & $14 \mathrm{~h}$ \\
\hline Hagg et al. ${ }^{13}$ & 30 & Disjuntor Palatino + Reverse Headgear Plus & $700 \mathrm{~g}$ & $45^{\circ}$ & $14 \mathrm{~h}$ \\
\hline Keles et al..$^{19}$ & 20 & Splint de acrílico + Máscara Facial / MPH & $500 \mathrm{~g}$ & $30^{\circ}$ & $16 \mathrm{~h}$ \\
\hline Baccetti et al. ${ }^{3}$ & 29 & Splint de acrílico + Máscara Facial de Petit & $400 \mathrm{~g}$ & Frente e p/baixo & $14 \mathrm{~h}$ \\
\hline Ngan, Yui ${ }^{28}$ & 20 & Hyrax + Máscara Facial & $380 \mathrm{~g}$ & $30^{\circ}$ & $12 \mathrm{~h}$ \\
\hline Suda et al. ${ }^{37}$ & 30 & Arco lingual + Reverse Pull Headgear & $215 \mathrm{~g}$ & $25^{\circ}$ & $10 \mathrm{~h}$ \\
\hline Saadia, Torres ${ }^{34}$ & 112 & Hyrax/Haas + Máscara Facial de Petit & $395 \mathrm{~g}$ & $30^{\circ}$ & $14 \mathrm{~h}$ \\
\hline Alcan, Keles, Erverdi ${ }^{1}$ & 17 & Disjuntor + Facebow + Máscara Facial de Delaire & $750 \mathrm{~g}$ & $\begin{array}{l}\text { Paralelo ao Plano de } \\
\text { Frankfurt }\end{array}$ & $17 \mathrm{~h}$ \\
\hline MacDonald et al. ${ }^{24}$ & 24 & Aparelho de Expansão + Máscara Facial & $325 \mathrm{~g}$ & $22,5^{\circ}$ & $20 \mathrm{~h}$ \\
\hline Yoshida et al. ${ }^{44}$ & 28 & Aparelho intrabucal + APM + Mentoneira & $250 \mathrm{~g}$ & $20^{\circ}$ & $14 \mathrm{~h}$ \\
\hline Franchi et al. ${ }^{11}$ & 46 & Aparelho de Expansão + Máscara Facial & $396 \mathrm{~g}$ & Frente e p/baixo & $22 \mathrm{~h}$ \\
\hline Ngan et al. ${ }^{29}$ & 20 & Hyrax + Máscara Facial & $380 \mathrm{~g}$ & $30^{\circ}$ & $12 \mathrm{~h}$ \\
\hline Gallagher et al. ${ }^{12}$ & 22 & Aparelho de Expansão + Máscara Facial & $700 \mathrm{~g}$ & Frente e baixo & $22 \mathrm{~h}$ \\
\hline Pangrazio-Kulbersh et al. ${ }^{32}$ & 40 & Disjuntor Palatino + Reverse Pull Headgear & $500 \mathrm{~g}$ & $30^{\circ}$ & $15 \mathrm{~h}$ \\
\hline Baccetti et al. ${ }^{4}$ & 46 & Splint de acrílico + Máscara Facial de Petit & $396 \mathrm{~g}$ & Frente e baixo & $22 h$ \\
\hline Sung, Baik ${ }^{38}$ & 129 & $\begin{array}{l}\text { Disjuntor/Aparelho labiolingual + Máscara Facial de } \\
\text { Delaire }\end{array}$ & $350 \mathrm{~g}$ & $25^{\circ}$ & $12 \mathrm{~h}$ \\
\hline Killiçoglu, Kirliç ${ }^{21}$ & 16 & Aparelho intrabucal + Máscara Facial de Delaire & $400 \mathrm{~g}$ & $22,5^{\circ}$ & $11 \mathrm{~h}$ \\
\hline Shanker et al. ${ }^{35}$ & 25 & Hyrax + Máscara Facial & $400 \mathrm{~g}$ & $30^{\circ}$ & $12 \mathrm{~h}$ \\
\hline Merwin et al. ${ }^{25}$ & 30 & Hyrax + Tubinger Reverse Pull Headgear & $380 \mathrm{~g}$ & $30^{\circ}$ & $13 \mathrm{~h}$ \\
\hline Ngan et al. ${ }^{27}$ & 30 & Hyrax + Máscara Facial & $380 \mathrm{~g}$ & $30^{\circ}$ & $12 \mathrm{~h}$ \\
\hline Chong, Ive, Artur ${ }^{8}$ & 16 & Aparelho intrabucal + Máscara Facial & $257,5 \mathrm{~g}$ & $35^{\circ}$ & $14 \mathrm{~h}$ \\
\hline Baik $^{6}$ & 60 & $\begin{array}{l}\text { Disjuntor Palatino / Ap. labiolingual + Máscara Facial } \\
\text { de Delaire }\end{array}$ & $400 \mathrm{~g}$ & Frente e baixo & $12 \mathrm{~h}$ \\
\hline Ngan et al. ${ }^{30}$ & 10 & Hyrax + Máscara Facial & $700 \mathrm{~g}$ & $45^{\circ}$ & $14 \mathrm{~h}$ \\
\hline
\end{tabular}


Somente 39 artigos foram selecionados após este processo seletivo. Os artigos incluídos foram aqueles que descreveram os três fatores analisados: magnitude da força, vetor de direção e horas de uso do aparelho de protração maxilar.

Os dados desses trabalhos foram organizados em uma tabela (Tab. 1) e foram obtidas as médias e os desvios-padrão, além dos valores mínimos e máximos, de cada estudo, para a magnitude da força, o vetor de direção e as horas diárias de uso do aparelho de protração maxilar.

\section{RESULTADOS}

As estratégias de busca e os resultados encontrados no Medline database - Entrez PubMed e Bireme são mostrados na figura 1 e no quadro 1 .

\section{Magnitude da força}

As médias e os desvios-padrão, bem como os valores mínimos e máximos para a magnitude da força, encontram-se na tabela 2.

A magnitude da força utilizada durante o processo de protração maxilar variou de acordo com cada estudo, indo desde a mínima força utilizada - de $180 \mathrm{~g}$ a $250 \mathrm{~g}$ (média $215 \mathrm{~g}$ ) relatada por Suda et al. ${ }^{37}$ - até a intensidade de $800 \mathrm{~g}$, aplicada no estudo de Kajiyama et al. ${ }^{15}$ Diversas variações na magnitude das forças aplicadas foram encontradas nos artigos selecionados.

TABELA 2 - Números de estudos com as seguintes informações: média, desvio-padrão (d.p.) e medidas mínima e máxima para a magnitude, a direção e a duração das forças utilizadas para a protração maxilar.

$\begin{array}{rccc} & \begin{array}{r}\text { Magnitude } \\ (\mathrm{g})\end{array} & \begin{array}{r}\text { Direção } \\ \text { (graus) }\end{array} & \begin{array}{c}\text { Duração } \\ (\mathrm{h} / \mathrm{d})\end{array} \\ \begin{array}{r}\text { Número de } \\ \text { artigos }\end{array} & 39 & 29 * & 39 \\ \text { Média } & 447,8 & 27,5 & 15,2 \\ \text { d.p. } & 148,5 & 6,6 & 3,5 \\ \text { Mínimo } & 215 & 20 & 10 \\ \text { Máximo } & 800 & 45 & 22\end{array}$

${ }^{*}$ Alguns estudos foram desconsiderados por não apresentarem o valor em graus dos vetores.
Magnitude até $300 \mathrm{~g}$

Suda et al. ${ }^{37}$ utilizaram uma força média de $215 \mathrm{~g}$, de forma similar a Weissheimer et al. . $^{42}$, que utilizaram, durante as duas primeiras semanas de tratamento, a força de $200 \mathrm{~g}$ a $250 \mathrm{~g}$ (média $225 \mathrm{~g}$ ). Chong et al. ${ }^{8}$ também usaram uma força moderada, variando de $230 \mathrm{~g}$ a $285 \mathrm{~g}$ (média 257,5g), semelhante à força de $200 \mathrm{~g}$ a $300 \mathrm{~g}$ (média $250 \mathrm{~g}$ ) mencionada em alguns estudos ${ }^{44,45,46}$ e a de $300 \mathrm{~g}$ relatada por Kircelli e Pektas ${ }^{22}$.

Magnitude de $300 \mathrm{~g}$ a $500 \mathrm{~g}$

A força de $380 \mathrm{~g}$ relatada por alguns autores $^{25,27,28,29}$ foi bem próxima da força de $395 \mathrm{~g}$ usada por Saadia e Torres ${ }^{34}$ e de $396 \mathrm{~g}$ utilizada por Baccetti et al. ${ }^{4}$ e Franchi et al. ${ }^{11}$ Já Sung e Baik ${ }^{38}$ variaram a força entre $300 \mathrm{~g}$ e $400 \mathrm{~g}$ (média 350g), como nos estudos que usaram a força de $400 \mathrm{~g}^{3,9,21,35}$. MacDonald, Kapust e Turley ${ }^{24}$ utilizaram uma força entre $200 \mathrm{~g}$ e $450 \mathrm{~g}$ (média $325 \mathrm{~g}$ ) e diversos autores afirmaram utilizar uma força entre $300 \mathrm{~g}$ e $500 \mathrm{~g}$ (média 400g) ) $^{5,610,41,43}$, próxima da empregada nos estudos que usaram $500 \mathrm{~g}$ de força $\mathrm{a}^{2,19,23,32,33}$.

Magnitude acima de $500 \mathrm{~g}$

Alguns autores relataram a força de $600 \mathrm{~g}^{16,31,40}$ e de $600 \mathrm{~g}$ a $700 \mathrm{~g}$ (média $650 \mathrm{~g}$ ) $^{20}$. Já em outros estudos, a força variou de $700 \mathrm{~g}^{12,13,30}$ a $750 \mathrm{~g}^{1}$. A força mais pesada aplicada para a protração maxilar relatada dentre os estudos selecionados foi de 800 g, usada por Kajiyama, Murakami e Suzuki ${ }^{15}$.

\section{Direção do vetor de força}

As médias e os desvios-padrão, bem como os valores mínimos e máximos para a direção do vetor de força, encontram-se na tabela 2.

A direção do vetor da força utilizada durante a protração maxilar apresentou variação entre os estudos selecionados. A maioria dos estudos utilizou o vetor para frente e para baixo, tendo como referência o Plano Oclusal, variando apenas o grau de inclinação. $\mathrm{O}$ único estudo que não utilizou o Plano Oclusal como referência foi o de Alcan et al. ${ }^{1}$, 
pois a força foi direcionada paralelamente ao Plano de Frankfurt.

Alguns estudos ${ }^{3-6,9-12,43}$ foram desconsiderados para a realização das médias e desvios-padrão, por não apresentarem o valor em graus dos vetores. Os autores relataram apenas o vetor para frente e para baixo em relação ao Plano Oclusal.

Vetor para frente e para baixo até $20^{\circ}$

Diversos estudos ${ }^{16,20,40,42,44,45,46}$ relataram o uso do vetor força com $20^{\circ}$ em relação ao Plano Oclusal.

Vetor para frente e para baixo $21^{\circ}$ a $30^{\circ}$

Para Vaughn et al. ${ }^{41}$ e MacDonald et al. ${ }^{24}$, a média da direção da força foi de $22,5^{\circ}$ em relação ao Plano Oclusal, similar à de Killiçoglu e Kirliç ${ }^{21}$, que usaram o vetor para frente e para baixo com a inclinação de $20^{\circ}$ a $25^{\circ}$ (média $22,5^{\circ}$ ). Outros estudos utilizaram $25^{\circ}$ em relação ao Plano Oclusal ${ }^{2,37,38}$.

$\mathrm{O}$ vetor de força de $30^{\circ}$ em relação ao Plano Oclusal foi o utilizado pelo maior número de estudos ${ }^{15,19,22,23,25,27,28,29,31-35}$.

Vetor para frente e para baixo acima de $30^{\circ}$

Apenas os estudos de Hagg et al. ${ }^{13}$ e Ngan et al. ${ }^{30}$ relataram uma direção de força para frente e para baixo de $45^{\circ}$ em relação ao Plano Oclusal.

\section{Tempo - duração diária de horas de uso}

As médias e os desvios-padrão, bem como os valores mínimos e máximos para o tempo diário de horas de uso do aparelho de protração maxilar, encontram-se na tabela 2.

As horas de uso do aparelho para promover a protração maxilar foram diferenciadas de acordo com cada autor.

\section{Até 12 horas/dia}

No estudo de Suda et al. ${ }^{37}$, foi orientado o uso do aparelho por no mínimo 10 horas ao dia. A instrução de uso diário de 10/12h (média 11h) e 12h foi preconizada por outros autores $\mathrm{s}^{6,15,21,23,27,28,29,35,38}$.

\section{De 13 a 16 horas/dia}

No estudo de Merwin et al. ${ }^{25}$, os pacientes foram orientados a usar o aparelho por um período médio de 13 horas. Outros trabalhos $2,3,5,9,10,34,43-46$ relataram o uso de, no mínimo, 14 horas por dia, semelhante à média de 14 horas relatada em outros estudos ${ }^{8,13,30}$. Para Pangrazio-Kulbersh, Berger, Kersten ${ }^{32}$ e Pangrazio-Kulbersh et al. ${ }^{33}$, as horas mínimas de uso variaram entre $14 \mathrm{~h}$ e $16 \mathrm{~h}$ (média 15h) por dia.

\section{Mais de 16 horas/dia}

Para alguns autores ${ }^{19,31,40}$, a recomendação para o uso do aparelho foi de 16 horas por dia, sendo que para Kama et al. ${ }^{16}$ a instrução foi de $16 \mathrm{~h}$ a $18 \mathrm{~h}$ (média 17h) e para Kilinç et al. ${ }^{20}$ de $18 \mathrm{~h}$. Alcan et al. ${ }^{1}$ sugeriram $17 \mathrm{~h}$ e McDonald et al. ${ }^{24}$ indicaram o tempo de $18 \mathrm{~h}$ a $22 \mathrm{~h}$ (média 20h) por dia. Alguns estudos recomendaram usar o aparelho de protração o máximo possível ${ }^{12}$ ou por tempo inte$\operatorname{gral}^{4,11,22,41,42}$, correspondente à média de $22 \mathrm{~h}$.

\section{DISCUSSÃO}

O tratamento da Classe III com protração maxilar tem sido bastante relatado na literatu$\mathrm{ra}^{1,2,12,21,24,30,32,40,41,43}$, porém a maioria dos trabalhos não identifica claramente a magnitude, a direção da força e o tempo de utilização do aparelho para obter-se a correção adequada desse problema. Nessa revisão sistemática, uma cuidadosa pesquisa da literatura foi realizada para encontrar estudos primários nos quais esses fatores fossem abordados.

Até o momento, apenas uma revisão sistemáti$\mathrm{Ca}^{39}$ e duas meta-análises foram encontradas, na literatura, sobre o tratamento da Classe III por meio da protração maxilar. Todavia, elas não avaliaram diretamente a magnitude das forças, os vetores de direção e as horas diárias de uso do aparelho.

Dos 39 estudos primários selecionados para a realização desse trabalho, apenas um artigo tratava-se de um ensaio clínico aleatório ${ }^{41}$, demonstrando, assim, a ausência de estudos com fortes evidências. 
Nos estudos selecionados, a magnitude mínima da força recomendada foi de $215 \mathrm{~g}^{37}$ e a máxima foi de $800 \mathrm{~g}^{15}$. A magnitude média determinada no presente trabalho foi de $447,8 \mathrm{~g}$, estando condizente com a maioria das recomendações dos demais estudos $23,3,5,6,9,10,19,21,23,32,33,35,42$.

Em todos os estudos selecionados, os autores demonstraram preocupação em evitar rotações indesejáveis da maxila durante o processo de protração maxilar. A direção do vetor de força para frente e para baixo apresentou inclinação mínima de 20 graus $^{16,20,39,41,43,44,45}$ e máxima de 45 graus $^{13,30}$ em relação ao Plano Oclusal. A inclinação média determinada neste trabalho foi de $27,5^{\circ}$ em relação ao Plano Oclusal, semelhante com a recomendação da maioria dos estudos ${ }^{15,19,22,23,25,27,28,29,31-35}$.

A quantidade de horas diárias de uso do aparetho de protração maxilar recomendada nos estudos foi de 10 horas/dia ${ }^{37}$ a 22 horas/dia 4 ,11,12,22,41. A média de horas/dia calculada no presente trabalho foi de 15,2 para o uso do aparelho, exatamente como recomendado por Pangrazio-Kulbersh, Berger, Kersten ${ }^{32}$ e Pangrazio-Kulbersh et al. ${ }^{33}$

Espera-se que os valores levantados, bem como as médias para a magnitude, a direção e o tempo diário da aplicação das forças, possam auxiliar aos profissionais da Ortodontia durante a realização da protração maxilar.

O presente estudo apresentou apenas os valores referidos pelos autores dos trabalhos selecionados, relativos às médias da magnitude, ao vetor de direção e ao tempo de uso das forças. Entretanto, o trabalho não avaliou os efeitos dentários e ou esqueléticos obtidos com essas prescrições de utilização do aparelho de protração, bem como as suas modificações, nem tampouco separou os efeitos da protração associada ou não à disjunção palatina mediana.
Novas pesquisas já estão sendo realizadas com o propósito de se verificar, especificamente, os resultados obtidos com essa terapia e as suas variações, bem como definir a recomendação mais adequada, tendo em vista resultados mais estáveis em longo prazo.

\section{CONCLUSÕES}

A protração maxilar, associada ou não à disjunção da sutura palatina mediana, é a terapia mais recomendada pelos autores para o tratamento da Classe III em fase de crescimento.

Por meio dessa revisão sistemática foram analisados os estudos primários que apresentaram os fatores magnitude, direção e duração diária da aplicação das forças de protração maxilar, sendo possível determinar que:

1) A média da magnitude da força de protração maxilar foi de 447,8g, com um desvio-padrão de $148,5 \mathrm{~g}$.

2) A média da inclinação do vetor da força de protração maxilar foi de 27,5 graus em relação ao Plano Oclusal, com um desvio-padrão de 6,6 graus.

3) A média do tempo de uso do aparelho de protração maxilar foi de 15,2 horas por dia, com desvio-padrão de 3,5 horas.

Novos estudos deverão ser realizados para avaliar os efeitos, na prática clínica, da aplicação das médias sugeridas e suas variações. 


\title{
The treatment of Class III - systematic review - Part I. Magnitude, direction and duration of the forces in the maxillary protraction
}

\begin{abstract}
Introduction: For the treatment of Class III during growth, maxillary protraction therapy is the most reported in the literature. During the treatment is required special attention to the mechanics used to fix the problem. Aim: To synthesize informations about the magnitude, direction and time of daily application of forces, through a systematic review. Methods: Studies were identified from an electronic search on Medline database - Entrez PubMed (178 articles) and Bireme (550 articles), from January 1983 until December 2008. After rigorous process of inclusion and exclusion 56 primary studies were selected and subjected to a second selection process, remaining 39 articles. It was calculated the average and standard deviation, and the minimum and maximum values for magnitude, direction and usage hours of the maxillary protraction forces. Results and Conclusion: The mean magnitude, direction and duration of the forces of maxillary protraction were, respectively: 447.8 grams, 27.5 degrees of inclination in relation to the occlusal plane and 15.2 hours per day.
\end{abstract}

Keywords: Angle Class III malocclusion. Orthopedic appliances. Orthodontics. Facial masks.

\section{REFERÊNCIAS}

1. ALCAN, T.; KELES, A.; ERVERDI, N. The effects of a modified protraction headgear on maxilla. Am. J. Orthod. Dentofacial Orthop., St. Louis, v. 117, no. 1, p. 27-38, Jan. 2000.

2. ARMAN, A.; TOYGAR, T. U.; ABUHULEH, E. Evaluation of maxillary protraction and fixed appliance therapy in Class III patients. Eur. J. Orthod., Oxford, v. 28, no. 4, p. 383-392, May 2006.

3. BACCETTI, T.; FRANCHI, L.; McNAMARA, J. A. Treatment and post treatment craniofacial changes after rapid maxillary expansion and facemask therapy. Am. J. Orthod. Dentofacial Orthop., St. Louis, v. 118, no. 4, p. 404-413, Oct. 2000.

4. BACCETTI, T.; McGILL, J. S.; FRANCHI, L.; McNAMARA, J. A.; TOLLARO, I. Skeletal effects of early treatment of Class III malocclusion with maxillary expansion and face-mask therapy. Am. J. Orthod. Dentofacial Orthop., St. Louis, v. 113, no. 3, p. 333-343, Mar. 1998

5. BACCETTI, T.; REY, D.; ANGEL, D.; OBERTI, G.; McNAMARA, J. A. Mandibular cervical headgear vs rapid maxillary expansion and facemask for orthopedic treatment of Class III malocclusion. Angle Orthod., Appleton, v. 77, no. 4, p. 619-624, Sept. 2006.

6. BAIK, H. S. Clinical results of the maxillary protraction in Korean children. Am. J. Orthod. Dentofacial Orthop., St. Louis, v. 108 , no. 6, p. 583-592, Dec. 1995

7. BAPTISTA, A. A.; CURY, S. A. A.; MOTTA, A. F. J.; VILELLA, O. $V_{\text {.; }}$ MUCHA, J. N. A prevalência de más-oclusões em escolares de Niterói. Rev. Flum. Odontol., Niterói, v. 2, n. 8, p. 34-41, maio/ago. 1998.

8. CHONG, Y. H.; IVE, J. C.; ARTUR, J. Changes following the use of protraction headgear for early correction of Class III malocclusion. Angle Orthod., Appleton, v. 66, no. 5, p. 351-362 1996.

9. COZZA, P.; MARINO, A.; MUCEDERO, M. An orthopedic approach to the treatment of Class III malocclusion in the early mixed dentition. Eur. J. Orthod., Oxford, v. 26, no. 2, p. 191-199, 2004.

10. FRANCHI, L.; BACCETTI, T.; McNAMARA, J. A. Postpubertal assessment of treatment timing for maxillary expansion and protraction therapy followed by fixed appliance. Am. J. Orthod. Dentofacial Orthop., St. Louis, v. 126, no. 5, p. 555-568, Nov. 2004
11. FRANCHI, L.; BACCETTI, T.; McNAMARA, J. A. Shape-coordinate analysis of skeletal changes induce by rapid maxillary expansion and facemask therapy. Am. J. Orthod. Dentofacial Orthop., St. Louis, v. 114, no. 4, p. 418-426, Oct. 1998.

12. GALLAGHER, R. W.; MIRANDA, F.; BUSCHANG, P. H. Maxillary protraction: Treatment and post treatment effects. Am. J. Orthod. Dentofacial Orthop., St. Louis, v. 113, no. 6, p. 612-619, June 1998.

13. HAGG, U.; TSE, A.; BENDEUS, M.; RABIE, A. B. M. Long-term follow-up of early treatment with reverse headgear. Eur. J. Orthod., Oxford, v. 25, no. 1, p. 95-102, Feb. 2003

14. HATA, S.; ITOH, T.; NAKAGAWA, M.; KAMOGASHIRA, K. ICHIKAWA, K.; MATSUMOTO, M.; CHACONAS, S. T. Biomechanical effects of maxillary protraction on the craniofacial complex. Am. J. Orthod. Dentofacial Orthop., St. Louis, v. 91, no. 4, p. 305-311, 1987.

15. KAJIYAMA, K.; MURAKAMI, T.; SUZUKI, A. Comparison of orthodontic and orthopedic effects of a modified maxillary protractor between deciduous and early mixed dentitions. Am. J. Orthod. Dentofacial Orthop., St. Louis, v. 126, no. 1, p. 23-32, July 2004

16. KAMA, D. J.; ÖZER, T.; BARAN, S. Orthodontic and orthopaedic changes associated with treatment in subjects with Class III malocclusion. Eur. J. Orthod., Oxford, v. 28, no. 5, p. 496-502, Oct. 2006.

17. KAMBARA, T. Dentofacial changes produced by extraoral forward force in the Macaca Irus. Am. J. Orthod., St. Louis, v. 71, no. 3, p. 249-277, 1977

18. KANG, H. K.; RYU, Y. K. A study on the prevalence of malocclusion of Yonsei University students in 1991. Korea J. Orthod. Seoul, v. 22, p. 3691-3701, 1992

19. KELES, A.; TOKMAK, E. C.; ERVERDI, N.; NANDA, R. Effect of varying the force direction on maxillary orthopedic protraction. Angle Orthod., Appleton, v. 72, no. 5, p. 387-396, Oct. 2002.

20. KILINÇ, A. S.; ARSLAN, S. G.; KAMA, J. D.; ÖZER, T.; DARI, O. Effects on the sagittal pharyngeal dimensions of protraction and rapid palatal expansion in Class III malocclusion subject. Eur. J. Orthod., Oxford, v. 30, no. 1, p. 61-66, 2008

21. KILLICCOGLU, H.; KIRLIC, Y. Profile changes in patients with class III malocclusion after Delaire mask face therapy. Am. J. Orthod. Dentofacial Orthop., St. Louis, v. 113, no. 4 p. 453-462, Apr. 1998 
22. KIRCELLI, B. H.: PEKTAS, Z. O. Midfacial protraction with skeletally anchored face mask therapy: A novel approach and preliminary results. Am. J. Orthod. Dentofacial Orthop., St. Louis, v. 133, no. 3, p. 440-449, Mar. 2008.

23. KYUNG-SUK, C. H. A. Skeletal changes of maxillary protraction in patients exhibiting skeletal Class III malocclusion: A comparison of three skeletal maturation groups. Angle Orthod. Appleton, v. 73, no. 1, p. 26-35, 2003.

24. MacDONALD, K. E.; KAPUST, A. J.; TURLEY, P. K. Cephalometric changes after the correction of Class III malocclusion with maxillary expansion: Facemask therapy. Am. J. Orthod. Dentofacial Orthop. St. Louis, v. 116, no. 1, p. 13-24, July 1999.

25. MERWIN, D.; NGAN, P.; HAGG, U.; YUI, C.; WEI, S. H. Y. Timing for effective application of anteriorly directed orthopedic force to the maxilla. Am. J. Orthod. Dentofacial Orthop., St. Louis, v. 112, no. 3, p. 292-299, Sept. 1997.

26. MULROW, C. D. Rationale for systematic reviews. Br. Med. J., London, v. 309, no. 6954, p. 597-599, 1994

27. NGAN, P.; HÄGG, U.; YUI, C.; MERWIN, D.; WEI, S. H. Y. Treatment response to maxillary expansion and protraction. Eur. J. Orthod., Oxford, v. 18, no. 1, p. 151-168, 1996

28. NGAN, P.; YUI, C. Evaluation of treatment and post treatment changes of protraction facemask treatment using the Par index. Am. J. Orthod. Dentofacial Orthop., St. Louis, v. 118, no. 4, p. 414-420, Oct. 2000.

29. NGAN, P.; YUI, C.; HU, A.; HÄGG, U.; WEI, S. H. Y.; GUNEL, E. Cephalometric and occlusal changes following maxillary expansion and protraction. Eur. J. Orthod., Oxford, v. 20, no. 3, p. 237-254, 1998.

30. NGAN, P.; WEI, S. H. Y.; HÄGG, U.; YUI, C. K. Y.; MERWIN, D.; STICKEL, B. Effect of protraction headgear on Class III malocclusion. Quintessence Int., Berlin, v. 23, no. 3, p. 197-207, 1992.

31. OKTAY, H.; ULUKAYA, E. Maxillary protraction appliance effect on the size of the upper airway passage. Angle Orthod., Appleton, v. 78, no. 2, p. 209-214, 2008.

32. PANGRAZIO-KULBERSH, V.; BERGER, J.; KERSTEN, G. Effects of protraction mechanics on the midface. Am. J. Orthod. Dentofacial Orthop., St. Louis, v. 114, no. 5, p. 484-491, Nov. 1998.

33. PANGRAZIO-KULBERSH, V.; BERGER, J. L.; JANISSE, F. N.; BAYIRLI, B. Long-term stability of Class III treatment: Rapid palatal expansion and protraction facemask vs LeFort I maxillary advancement osteotomy. Am. J. Orthod. Dentofacial Orthop., St. Louis, v. 131, no. 1, p. 7.e9-e19, Jan. 2007.

34. SAADIA, M.; TORRES, E. Sagittal changes after maxillary protraction with expansion in Class III patients in the primary mixed and late mixed dentitions: A longitudinal retrospective study. Am. J. Orthod. Dentofacial Orthop., St. Louis, v. 117, no. 6, p. 669-680, June 2000.
35. SHANKER, S.; NGAN, P.; WADE, D.; BECK, M.; YIU, C.; HÄGG, U.; WEl, S. H. Y. Alterações cefalométricas do ponto A durante e após a expansão e protração maxilar. R. Dental Press Ortodon. Ortop. Facial, Maringá, v. 2, n. 6, p. 46-48, nov./dez. 1997.

36. SILVA, R. G.; KANG, D. S. Prevalence of malocclusion among latino adolescents. Am. J. Orthod. Dentofacial Orthop., St. Louis, v. 119, no. 3, p. 313-315, 2001

37. SUDA, N.; ISHII-SUZUKI, M.; HIROSE, K.; HIYAMA, S.; SUZUKI S.; KURODA, T. Effective treatment plan for maxillary protraction: Is the bone age useful to determine the treatment plan? Am. J. Orthod. Dentofacial Orthop., St. Louis, v. 118, no. 1, p. 55-62, July 2000 .

38. SUNG, S. J.; BAIK, H. S. Assessment of skeletal and dental changes by maxillary protraction. Am. J. Orthod. Dentofacial Orthop., St. Louis, v. 114, no. 5, p. 492-502, Nov. 1998.

39. TOFFOL, L.; PAVONI, C.; BACCETTI, T.; FRANCHI, L.; COZZA P. Orthopedic treatment outcomes in Class III malocclusion: A systematic review. Angle Orthod., Appleton, v. 78, no. 3, p. 561-573, 2007.

40. TORTOP, T.; KEYKUBAT, A.; YUKSEL, S. Facemask therapy with and without expansion. Am. J. Orthod. Dentofacial Orthop., St. Louis, v. 132, no. 4, p. 467-474, Oct. 2007.

41. VAUGHN, G. A.; MASON, B.; MOON, H. B.; TURLEY, P. K. The effects of maxillary protraction therapy with or without rapid palatal expansion: A prospective, randomized clinical trial

Am. J. Orthod. Dentofacial Orthop., St. Louis, v. 128, no. 3, p. 299-309, Sept. 2005.

42. WEISSHEIMER, F.; BRUNETTO, A. R.; PETRELLI, E. Disjunção palatal e protração maxilar: alterações cefalométricas pós-tratamento. J. Bras. Ortodon. Ortop. Facial, Curitiba, v. 8 n. 44, p. 111-121, mar./abr. 2003.

43. WESTWOOD, P. V.; MCNAMARA, J. A.; BACCETTI, T.; FRANCHI, L.; SARVER, D. M. Long-term effects of Class III treatment with rapid maxillary expansion and facemask therapy followed by fixed appliances. Am. J. Orthod. Dentofacial Orthop. St. Louis, v. 123, no. 3, p. 306-320, Mar. 2003.

44. YOSHIDA, I.; ISHII, H.; YAMAGUCHI, N.; MIZOGUCHI, I. Maxillary protraction and chin cap appliance treatment effects and long-term changes in skeletal Class III patients. Angle Orthod., Appleton, v. 69, no. 6, p. 543-552, 1999.

45. YOSHIDA, I.; SHOJI, T.; MIZOGUCHI, I. Effects of treatment with a combined maxillary protraction and chincap appliance in skeletal Class III patients with different vertical skeletal morphologies. Eur. J. Orthod., Oxford, v. 29, no. 2, p. 126-133, 2007.

46. YOSHIDA, I.; YAMAGUCHI, N.; MIZOGUCHI, I. Prediction of post-treatment outcome after combined treatment with maxillary protraction and chincap appliances. Eur. J. Orthod., Oxford, v. 28, no. 1, p. 89-96, 2006.
Endereço para correspondência

Anna Paula Rocha Perrone

Rua Duque de Caxias, no. 01, Sala 43 - Centro

CEP: 28.613-060 - Nova Friburgo / RJ

E-mail: apaularocha@uol.com.br 